\title{
Zuckerkandl Tubercle of the Thyroid Gland: Correlations between Findings of Anatomic Dissections and CT Imaging
}

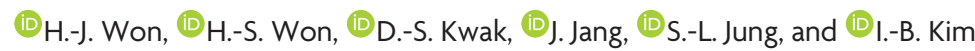

\begin{abstract}
BACKGROUND AND PURPOSE: The Zuckerkandl tubercle is located at the posteromedial border of the thyroid lobe, and it may be confused with a neoplasm or other mass. This study was performed to clarify the position and morphologic characteristics of the Zuckerkandl tubercle by dissecting cadavers and to compare the findings with the corresponding CT images obtained in the same cadavers.
\end{abstract}

MATERIALS AND METHODS: One hundred thyroid lobes from 50 fresh cadavers were dissected for this study ( 20 males and 30 females; mean age at death, $77.3 \pm 11.5$ years). CT scans were obtained in 10 of the cadavers by using a 128-channel multidetector row CT scanner before dissection.

RESULTS: The Zuckerkandl tubercle of the thyroid gland was observed in $83 \%$ of the specimens. It was mostly located at the posteromedial border of the thyroid lobe and within the middle two quarters (2nd and 3rd) of the thyroid lobe. The Zuckerkandl tubercle was classified into 3 types based on its direction of extension: posteromedial in $64 \%$ of the specimens, posteromedial and superior in $13 \%$, and posteromedial and inferior in $6 \%$. On axial CT, the Zuckerkandl tubercle was usually continuous with the posteromedial part of the thyroid lobe and extended posteromedially to the esophagus. The parts of the Zuckerkandl tubercle that protrude posteromedially and superiorly or posteromedially and inferiorly from the thyroid lobe appeared separated from the thyroid gland by a thin, low-density string on axial CT.

CONCLUSIONS: Zuckerkandl tubercles that protrude toward the posteromedial and superior or inferior direction could cause confusion due to their separation when performing diagnoses with CT images.

ABBREVIATION: ZT = Zuckerkandl tubercle

Z uckerkandl ${ }^{1}$ described a posterior projection of the thyroid lobe as the Zuckerkandl tubercle (ZT). The anatomic correlations of the ZT with the recurrent laryngeal nerve and the superior parathyroid gland were reported by Gilmour, ${ }^{2}$ but this structure subsequently received little attention. Following the description of the ZT as a constant anatomic landmark of the recurrent laryngeal nerve and its classification into 4 grades according to its size, ${ }^{3}$ the topographic anatomy of these structures and the grading of the ZT have been studied both in patients during surgery $^{4-8}$ and in cadavers. ${ }^{9,10}$ ZT has mostly been defined as a pos-

Received September 7, 2016; accepted after revision February 9, 2017. From the Department of Anatomy (H.-J.W., H.-S.W., D.-S.K., I.-B.K.), Catholic Institute for Applied Anatomy, College of Medicine, The Catholic University of Korea, Seoul, Korea; Department of Mortuary Science (H.-J.W.), Eulji University, Seongnam, Korea; Department of Anatomy (H.-S.W.), School of Medicine, Wonkwang University, Iksan, Korea; and Department of Radiology (I.J., S.-L.J.), Seoul St. Mary's Hospital, The Catholic University of Korea, Seoul, Korea.

Please address correspondence to In-Beom Kim, MD, PhD, Department of Anatomy, Catholic Institute for Applied Anatomy, College of Medicine, The Catholic University of Korea, Banpo-daero, Seocho-gu, Seoul 06591, Korea; e-mail: ibkimmd@catholic.ac.kr

http://dx.doi.org/10.3174/ajnr.A5172 terior or lateral projection or a projection from the posterolateral border of the thyroid lobe in previous studies, but it was recently reported as a posteromedial projection in a normal anatomic position. ${ }^{11}$ The lateral lobe of the thyroid gland is medially retracted to find the ZT during the surgery, which could be responsible for the differences in the position and shape of the $\mathrm{ZT}$ relative to a previous study that investigated fresh cadavers. ${ }^{11}$

Most ZTs extend into the tracheoesophageal groove or posterior to the esophagus. ${ }^{8,11}$ Failing to remove the ZT in its entirety during total thyroidectomy may result in persistent unrelieved symptoms or recurrence $e^{4,12,13}$ as well as persistent radioiodine uptake on radioactive iodine scans. ${ }^{7}$ An enlarged ZT may increase pressure symptoms. ${ }^{4,14,15}$ Sonography is widely used for thyroid imaging, but CT is performed for nodules or nodule-like regions of the thyroid gland. ZT may be mistaken for a mass or lymph node on CT based on its morphology, resulting in unnecessary biopsies or additional imaging being performed. ${ }^{13}$

The aims of this study were to clarify the position and morphologic characteristics of the ZT by performing cadaver dissec- 


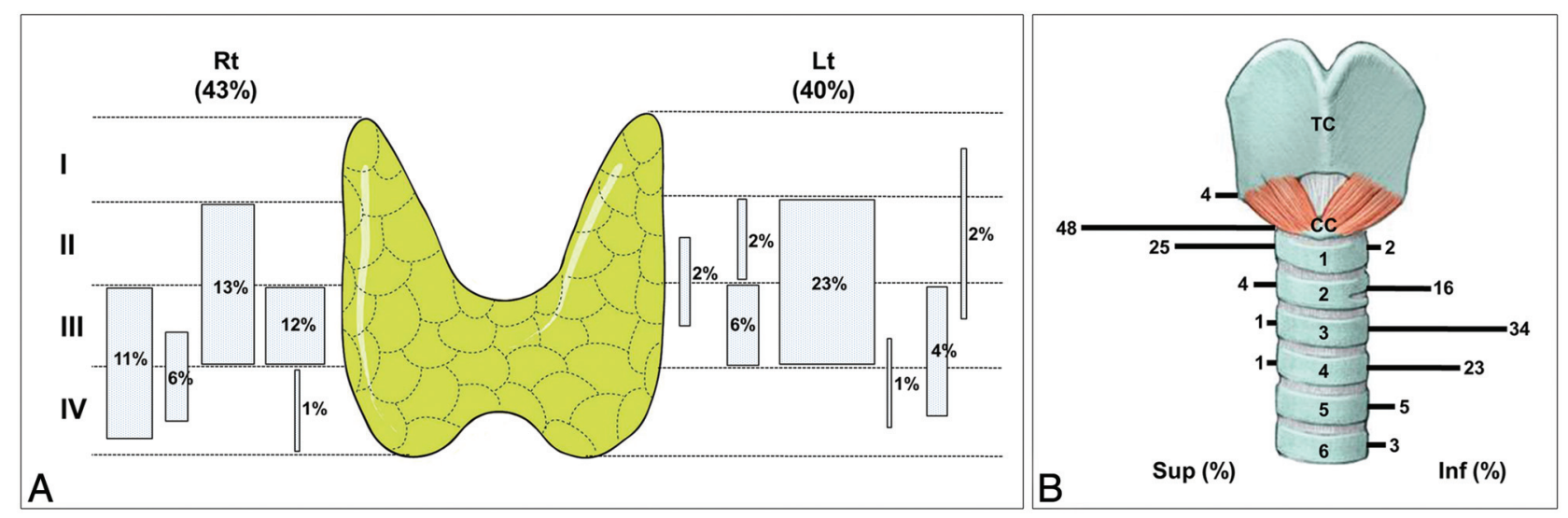

FIG 1. Location of the ZT and its superior and inferior borders according to the thyroid lobe $(A)$ and the level of the tracheal rings $(B)$. CC indicates cricoid cartilage; TC, thyroid cartilage; Rt, right; Lt, left; Sup, superior; Inf, inferior.

tions and to compare these findings with CT images obtained in the same cadavers.

\section{MATERIALS AND METHODS Cadavers}

One hundred thyroid lobes from 50 fresh Korean cadavers were used for this study (20 males and 30 females; mean age at death, 77.3 years; age range, $51-103$ years), and 10 of these cadavers were also used for CT imaging before dissection. No trauma-related morphologic changes were observed in the specimens during the dissection, and the medical histories related to thyroid disease were unknown. Examination of the external appearance of the thyroid glands revealed no enlargement, and pressure with the thumb and index finger was applied to all ZTs for detecting nodules. Due to the domestic law that cadavers must be donated and managed under an informed consent and ethical process, the institutional review board of our university has decreed that studies involving cadavers do not need to be reviewed. All cadavers stored at Catholic Institute for Applied Anatomy qualified as materials for education and research according to the domestic law.

\section{Neck CT Scanning}

CT was performed by using a 128-channel multidetector row CT scanner (Somatom Definition AS; Siemens, Erlangen, Germany) at 1-3 days after death. The neck of each cadaver was positioned in the center of the coil gantry in a neutral position, and axial images with a section thickness of $0.8 \mathrm{~mm}$ were obtained with following parameters: FOV, $200 \times 200 \mathrm{~mm}^{2}$; matrix, $512 \times 512$; tube voltage, $150 \mathrm{kV}$ (peak); tube current-time product $=160 \mathrm{mAs}$. Two radiologists specializing in head and neck radiology read the CT images. One is a professor with 30 years' experience in clinical diagnosis and research in head and neck radiology, and the other is a clinical fellow with 10 years' experience in clinical diagnosis and research.

\section{Dissection}

Thyroid glands were exposed after skin, platysma, and the sternocleidomastoid and infrahyoid muscles were sequentially removed. Then, each thyroid gland was removed with the trachea and esophagus en bloc by cutting above the thyroid cartilage, and it was immersed in fixative that included $5 \%$ formalin for 5 days. This produced a soft-state thyroid gland, which was then dissected.
The borders of the thyroid lobe and the direction of the projected part of the ZT were observed in their normal anatomic positions. Four thyroid glands with the trachea and esophagus were serially sectioned transversely at 2-mm intervals, and 2 of these were stained with hematoxylin-eosin to allow comparisons of the ZT tissues and those of the thyroid lobes. Cadaver dissection was performed by 5 anatomists specializing in human gross anatomy, and one of them, also specializing in histology, confirmed the histologic images. Three anatomists, including the corresponding author, are professors, and the other 2 anatomists are research fellows in the Department of Anatomy. All of the anatomists involved in the dissection have 10 to 50 years' experience in anatomic research. The pathologic conditions of the ZTs were confirmed by a pathologist, also a professor in the Department of Pathology, who has a board certification in pathology in the Korean Medical Association.

\section{RESULTS}

\section{Dissected Specimens}

The ZT of the thyroid gland was observed in $83 \%$ of the 100 thyroid lobes (40\% left and 43\% right; 34\% male and 49\% female), and it had no nodule. The ZT was located at the posteromedial border of the thyroid lobe in 77 specimens; in the other 6 specimens, it was identified at the posterior surface near the posteromedial border of the thyroid gland. The locations of the ZT and its superior and inferior borders was checked relative to the thyroid lobe (Fig 1A) and the level of the tracheal rings, respectively (Fig 1B). When the full longitudinal length of the thyroid lobe was divided equally into 4 parts, the ZT was found in $\geq 2$ parts in $62 \%$ of the specimens: $2 \%$ in parts I-III, $38 \%$ in parts II and III, and $22 \%$ in parts III and IV. The ZT was located only in parts II, III, and IV in $2 \%, 18 \%$, and $1 \%$ of the specimens, respectively. The superior border of the ZT was most frequently located on the inferior border of the cricoid cartilage ( $48 \%$ of the specimens), followed by the first tracheal ring (25\%). The inferior border of the ZT was most frequently found at the level of the third tracheal ring (34\%), followed by the fourth $(23 \%)$ and second $(16 \%)$ tracheal rings.

The ZT was classified into 3 types based on its direction of extension: posteromedial in $64 \%$ ( $31 \%$ left and 33\% right), posteromedial and superior in $13 \%$ ( $9 \%$ left and $4 \%$ right), and posteromedial and inferior in $6 \%$ (only on right side) of the specimens (Fig 2). The long axes of the posteromedially and superiorly or inferiorly extended ZTs 

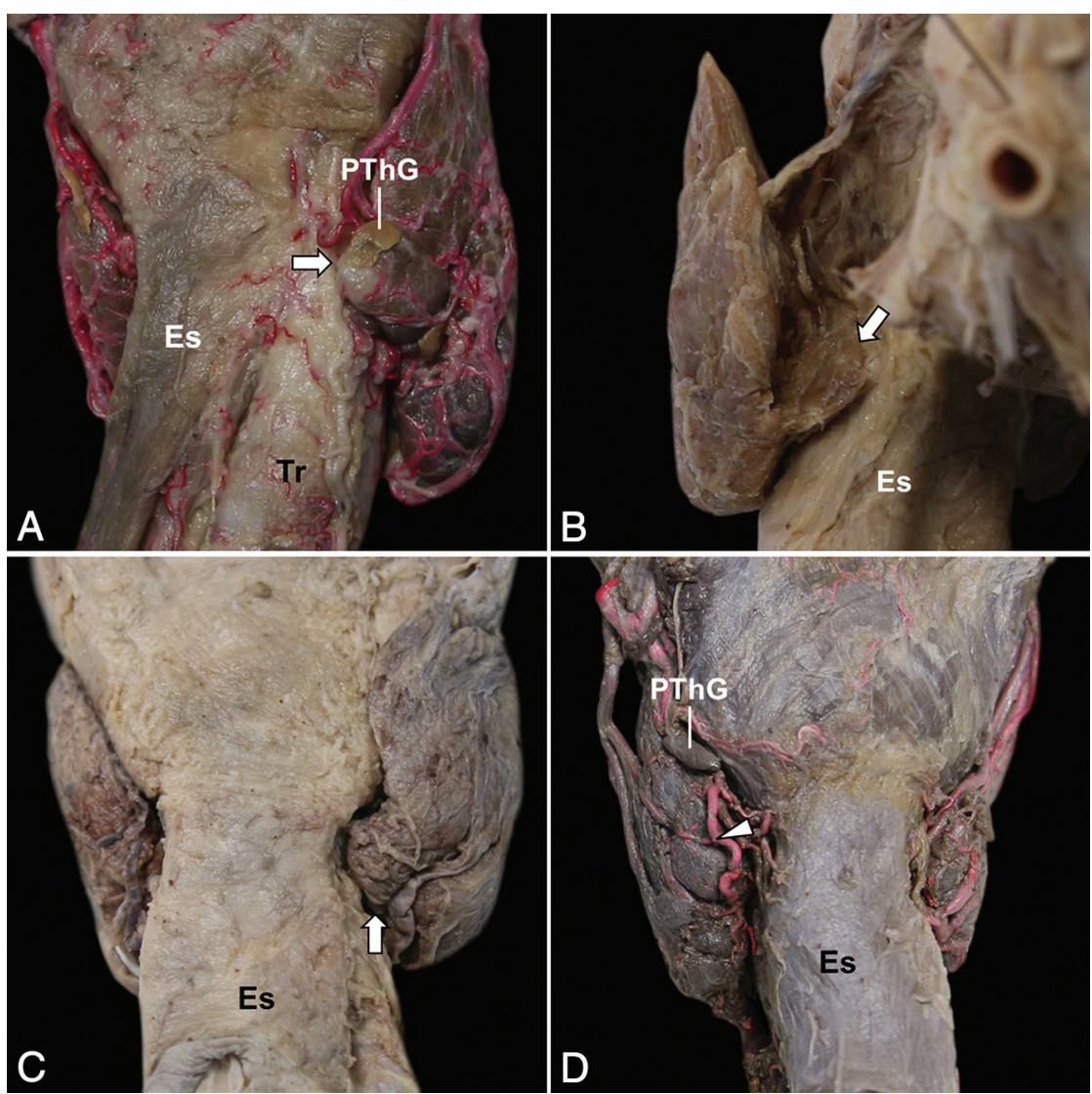

FIG 2. Types of ZTs based on the direction of extension: posteromedial $(A)$, posteromedial and superior $(B)$, and posteromedial and inferior (C). D, A specimen with an uneven ZT surface, which divides into 2 parts due to a deep groove (arrowhead) representing impressions of the blood vessels. Arrows indicate the ends of ZT extensions. Es indicates esophagus; PThG, parathyroid gland; Tr, trachea.

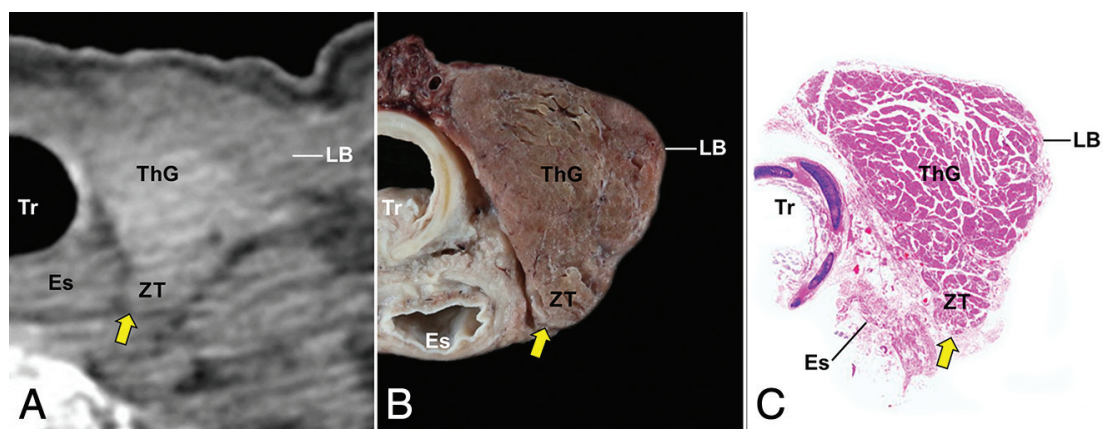

FIG 3. Comparison of ZT on axial CT (A), cross-sectional ( $B)$, and histologic $(C)$ images of the same specimen. The ZT (arrows) is continuous with the posterior part of the thyroid lobe, extends posteromedially toward the posterior end of the esophagus, and is compatible with the other areas of thyroid tissue in the microscopic finding. LB indicates lateral border of the thyroid lobe; Es, esophagus; ThG, thyroid gland; Tr, trachea.

were angled at a mean of $20.0^{\circ} \pm 11.6^{\circ}$ and $24.2^{\circ} \pm 13.1^{\circ}$ relative to the thyroid lobes, respectively. The surface of the $\mathrm{ZT}$ was uneven in $22 \%$ of the specimens, which had 1 (14\%), 2 (7\%), and 3 (1\%) deep grooves representing impressions of the blood vessels (Fig 2D). The microscopic findings of ZTs were compatible with the other areas of thyroid tissue (Fig 3C).

\section{CT Findings}

The ZT was observed in 14 of 20 thyroid lobes on axial CT images and was similarly identified in all the specimens when dissected.
The ZT was usually continuous with the posterior part of the thyroid lobe and extended posteromedially $(n=10)$ toward the tracheoesophageal groove or posterior end of the esophagus (Fig 3). Cases in which the ZT extended to the posteromedial and superior $(n=3)$ or inferior $(n=1)$ direction from the thyroid lobe were also detected in the series of axial CT images (Fig 4). When the ZT extended posteromedially and superiorly, it could be seen separately from the thyroid lobe in the upper level of the CT image, with the space between these structures appearing as a thin, low-density string. This string was not evident on the lower level of the CT image, where the ZT root merged with a thyroid lobe. When the ZT extended posteromedially and inferiorly, both it and the thyroid lobe were evident continuously in the upper level of the CT image, while the thin, low-density string appeared between them in the lower level of the CT image.

\section{DISCUSSION}

Most studies have found the incidence of ZT to lie within the range of 59\%$87 \%,{ }^{3,4,6,7,9-11,14,16,17}$ but there is 1 report of a very low incidence of $7 \% .{ }^{18}$ Most investigators have detected the ZT more frequently in the right thyroid lobe. ${ }^{7,16,17,19,20}$ In the present study, the ZT was identified in $83 \%$ of the specimens, which is consistent with the incidence range reported by most authors, and it was also observed more frequently on the right side but with no sex difference.

The lateral lobe of the thyroid gland is retracted medially during thyroid surgery to reveal the ZT in the lateral aspect. Thus, the ZT might have been regarded as a lateral projection or a projection from the posterolateral border of the thyroid lobe for a long time. $3,4,16,19$ However, its shape and position can differ according to the extent of thyroid lobe retraction and in excised thyroid lobes because of the softness of the thyroid gland. ${ }^{11}$ No lateral projection could be seen in previous anatomic studies reporting morphologic variations of the anterior view of the thyroid gland involving 60 white, ${ }^{21} 105$ Indian, ${ }^{22}$ and 168 Korean $^{23}$ cadavers. Recently, Won et al ${ }^{11}$ proposed descriptive terms for more accurate portrayal of the anatomic location and orientation of the ZT. The lateral border of the thyroid gland was defined as the most lateral margin of the anterior aspect of the thyroid in the anatomic position. The posteromedial border was defined as the margin 


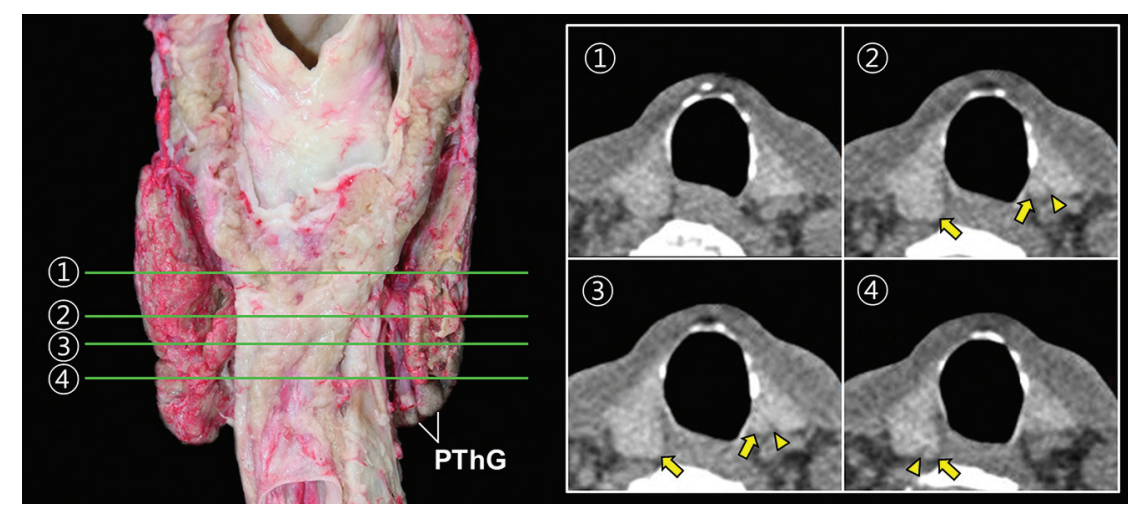

FIG 4. ZTs protruding posteromedially and superiorly (left side) and posteromedially and inferiorly (right side) in both thyroid lobes of the same cadaver, and their shapes on axial CT images at different levels. Arrowheads and arrows indicate the ZT and the slit between the ZT and the thyroid lobe, respectively. PThG indicates parathyroid gland.
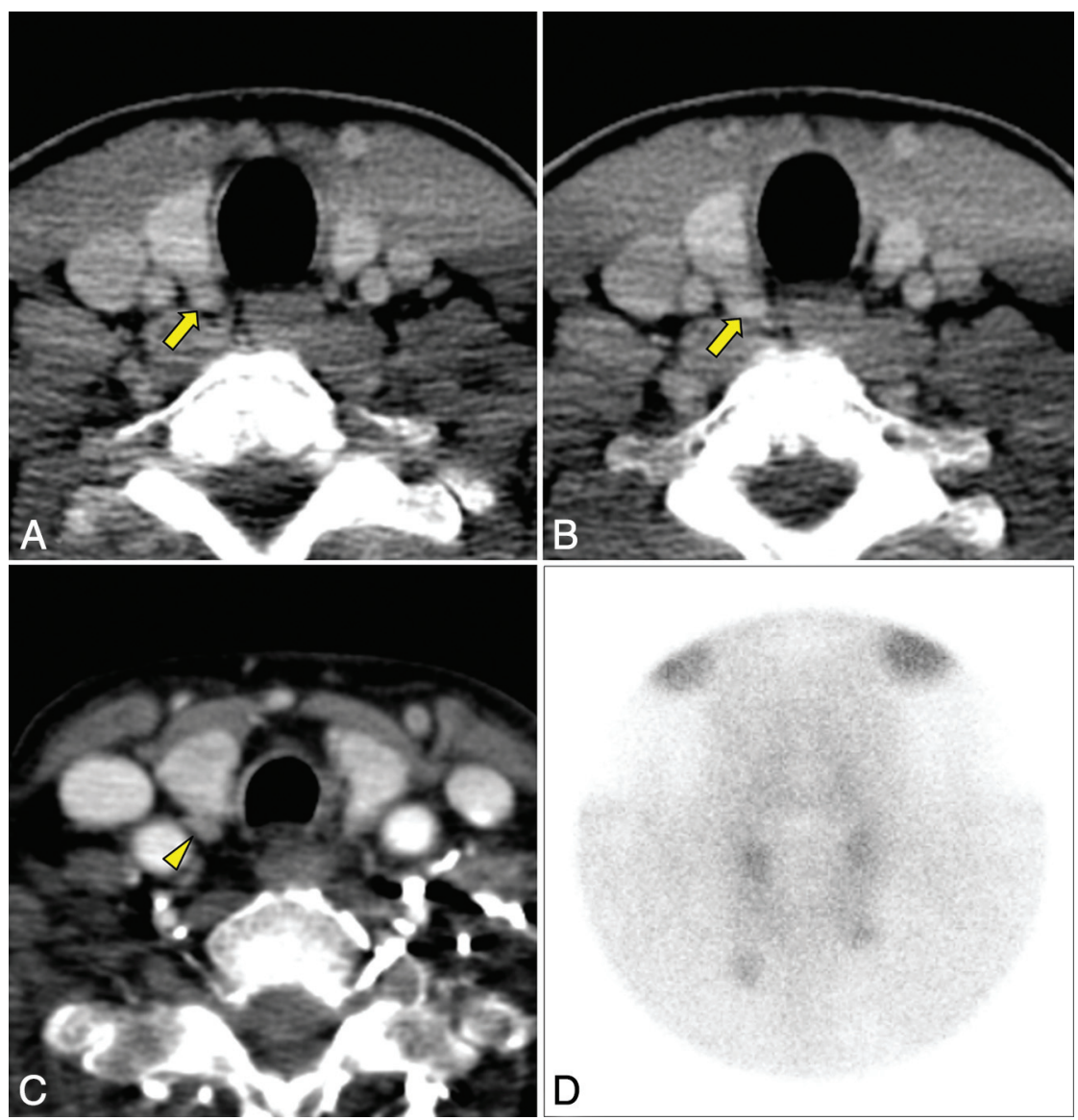

FIG 5. Comparison of clinically acquired axial CT images between the ZT appearing discontinuous from the thyroid gland and as a separate nodule or lymph node. A, Contrast-enhanced CT scan of a 33-year-old male patient who had undergone neck CT for work-up of lymphoma. There is a bulging structure (arrow) at the posterior aspect of the right thyroid lobe, and a fat plane between this structure and the thyroid lobe. The bulging structure protrudes toward the posterior and medial direction. The fat plane is not evident at the lower level of the CT image $(B)$, which means that the bulging structure is a ZT protruding in the posteromedial and superior direction. C, Contrast-enhanced CT scan of a 65-year-old female patient who had hyperparathyroidism. An oval nodular lesion (arrowhead) separated from the thyroid gland with a fat plane is seen posterior to the right thyroid lobe and is protruding in the posterior and lateral direction. This nodule is suggested to be a parathyroid adenoma or hyperplasia in the delayed image of the parathyroid scan (D). that exhibits the greatest projection toward the trachea or esophagus. This formed an angle on CT images through the isthmus of the thyroid gland. The posterior surface was between the lateral and posteromedial borders of the thyroid lobe. The posterolateral border was identifiable only in cases of compression by the common carotid artery in transverse sections of fixed cadavers and CT images. According to these terms, the ZT was mostly identified as a posterior or posteromedial projection at the posteromedial border of the thyroid lobe in a normal anatomic position. In the present study, the ZT also was not observed in the anterior view of the thyroid gland and was located at the posteromedial border of the thyroid lobe or posterior surface near the posteromedial border of the thyroid lobe.

The thyroid lobes are approximately conical and about $5 \mathrm{~cm}$ long. Their ascending apices diverge laterally to the level of the oblique lines on the laminae of the thyroid cartilage, and their bases are at the level of the fourth or fifth tracheal cartilage. ${ }^{24}$ The ZT was usually found at the middle third of the thyroid lobe in previous studies ${ }^{9,16}$ but was mainly observed within the middle two quarters (2nd and 3rd) of the full longitudinal length of the thyroid lobe in the present study (Fig 1A). Its superior border was mainly located on the inferior border of the cricoid cartilage (48\%), whereas its inferior border was diversely found at the levels of the second (16\%), third (34\%), and fourth (23\%) tracheal rings (Fig $1 B$ ).

The shape of the ZT was previously described as sessile (56.4\%) or pedunculated $(23.1 \%)$ in 195 dissected thyroid lobes gained from 107 thyroidectomies, ${ }^{16}$ and the nodular-shaped ZT having a narrow neck or stalk from the posterior border of the thyroid lobe was reported as the most common, occurring in more than one-third of the $\mathrm{CT}$ scans of 96 patients. ${ }^{13}$ ZT has previously been defined on axial CT images as the part of the thyroid gland extending posteriorly to the tracheoesophageal groove, and the nodular-shaped ZT could appear discontinuous from the thyroid gland. When the ZT had a nodular shape and particularly when the low 
neck was compromised by streak artifacts from the shoulders, it could be mistaken for a mass or lymph node. ${ }^{13}$

ZT can also be confused with a parathyroid adenoma, especially in patients with primary hyperparathyroidism. In a previous report reviewing primary hyperparathyroidism, ${ }^{25}$ there were some cases in which the findings of a diagnostic imaging study such as $\left[{ }^{11} \mathrm{C}\right]$ methionine PET/CT were positive but the surgeon could not locate the adenoma during the operation. We believe that some of these cases might have resulted from confusion of the ZTs with parathyroid adenomas. Theoretically, ZT has been described as the anatomic structure that separates the parathyroid glands into the superior and inferior parathyroid. ${ }^{3}$ Additionally, a previous report showed that the abnormal inferior parathyroid glands were more common than the abnormal superior glands in 810 parathyroidectomies reviewed. ${ }^{26}$ We believe that these anatomic characteristics and statistical results should be considered when examining for parathyroid adenoma in patients with primary hyperparathyroidism.

In the present study, ZT usually appeared continuous with the posterior part of the thyroid lobe on axial CT and extended posteromedially toward the tracheoesophageal groove or posterior end of the esophagus (Fig 3B). ZT was also confirmed as being one part of the thyroid gland pathohistologically (Fig 3C), which implies that the ZT can be identified by gross inspection. However, none of the ZTs had a stalk, and they all protruded directly from the thyroid lobe. ZT extending to the posteromedial and superior or inferior direction could only appear discontinuous from the thyroid gland on axial CT images (Figs 4 and $5 A$ ); this appearance might result in it being confused with a separate nodule, lymph node, or parathyroid adenoma (Fig 5C).

\section{CONCLUSIONS}

This study has revealed the diverse positions and morphologies of the ZT based on anatomic dissections, which are also confirmed in the corresponding CT images. The information obtained in this study is expected to be helpful for reducing confusion between the ZT and other structures when performing diagnoses with CT.

\section{ACKNOWLEDGMENTS}

The authors thank Professor In-Hyuk Chung in the Department of Anatomy, Yonsei University College of Medicine, and $\mathrm{Mr}$ Seong-In John for their help in dissecting the specimens and preparing the figures. Also, the authors thank Professor Soo Young Kim in the Department of Pathology, The Catholic University of Korea, for confirming pathologic conditions of the specimens.

\section{REFERENCES}

1. Zuckerkandl E. Nebst Bemerkungen über die Epithelkörperchen des Menschen. Anat Hefte 1902;LXI:61-82

2. Gilmour JR. The gross anatomy of the parathyroid glands. J Pathol Bacteriol 1938;46:133-49

3. Pelizzo MR, Toniato A, Gemo G. Zuckerkandl's tuberculum: an arrow pointing to the recurrent laryngeal nerve (constant anatomical landmark). J Am Coll Surg 1998;187:333-36 CrossRef Medline

4. Gauger PG, Delbridge LW, Thompson NW, et al. Incidence and importance of the tubercle of Zuckerkandl in thyroid surgery. Eur J Surg 2001;167:249-54 CrossRef Medline
5. Hisham AN, Lukman MR. Recurrent laryngeal nerve in thyroid surgery: a critical appraisal. ANZ J Surg 2002;72:887-89 CrossRef Medline

6. Yun JS, Lee YS, Jung JJ, et al. The Zuckerkandl's tubercle: a useful anatomical landmark for detecting both the recurrent laryngeal nerve and the superior parathyroid during thyroid surgery. Endocr J 2008;55:925-30 CrossRef Medline

7. Sheahan P, Murphy MS. Thyroid tubercle of Zuckerkandl: importance in thyroid surgery. Laryngoscope 2011;121:2335-37 CrossRef Medline

8. Gil-Carcedo E, Menéndez ME, Vallejo LA, et al. The Zuckerkandl tubercle: problematic or helpful in thyroid surgery? Eur Arch Otorhinolaryngol 2013;270:2327-32 CrossRef Medline

9. Yalçin B, Ozan H. Relationship between the Zuckerkandl's tubercle and entrance point of the inferior laryngeal nerve. Clin Anat 2007; 20:640-43 CrossRef Medline

10. Kaisha W, Wobenjo A, Saidi H. Topography of the recurrent laryngeal nerve in relation to the thyroid artery, Zuckerkandl tubercle, and Berry ligament in Kenyans. Clin Anat 2011;24:853-57 CrossRef Medline

11. Won HS, Liu HF, Kim JH, et al. Zuckerkandl's tubercle of the thyroid gland: its location in the anatomical position, and comparative morphology of the same specimens before and after fixation. Clin Anat 2015;28:472-76 CrossRef Medline

12. Reeve TS, Delbridge L, Cohen A, et al. Total thyroidectomy: the preferred option for multinodular goiter. Ann Surg 1987;206: 782-86 CrossRef Medline

13. Lee TC, Selvarajan SK, Curtin H, et al. Zuckerkandl tubercle of the thyroid: a common imaging finding that may mimic pathology. AJNR Am J Neuroradiol 2012;33:1134-38 CrossRef Medline

14. Hisham AN, Aina EN. Zuckerkandl's tubercle of the thyroid gland in association with pressure symptoms: a coincidence or consequence? Aust N Z J Surg 2000;70:251-53 CrossRef Medline

15. Delbridge L. Total thyroidectomy: the evolution of surgical technique. ANZ J Surg 2003;73:761-68 CrossRef Medline

16. Gil-Carcedo Sañudo E, Menéndez Argüelles ME, Vallejo Valdezate LÁ, et al. Zuckerkandl's tubercle. Location, shape and dimensions [in Spanish]. Acta Otorrinolaringol Esp 2012;63:443-49 CrossRef Medline

17. Irkorucu O. Zuckerkandl tubercle in thyroid surgery: is it a reality or a myth? Ann Med Surg (Lond) 2016;7:92-96 CrossRef Medline

18. Page C, Cuvelier P, Biet A, et al. Thyroid tubercle of Zuckerkandl: anatomical and surgical experience from 79 thyroidectomies. $J$ Laryngol Otol 2009;123:768-71 CrossRef Medline

19. Mehanna R, Murphy MS, Sheahan P. Thyroid tubercle of Zuckerkandl is more consistently present and larger on the right: a prospective series. Eur Thyroid J 2014;3:38-42 CrossRef Medline

20. Rajapaksha A, Fernando R, Ranasinghe N, et al. Morphology of the tubercle of Zuckerkandl and its importance in thyroid surgery. Ceylon Med J 2015;60:23-24 CrossRef Medline

21. Marshall CF. Variations in the form of the thyroid gland in man. $J$ Anat Physiol 1895;29(Pt 2):234-39 Medline

22. Ranade AV, Rai R, Pai MM, et al. Anatomical variations of the thyroid gland: possible surgical implications. Singapore Med J 2008;49: 831-34 Medline

23. Won HS, Chung IH. Morphologic variations of the thyroid gland in Korean adults [In Korean with English abstract and explanation of figures]. Korean J Phy Anthropol 2002;15:119-25

24. Gray H, Williams PL, Bannister LH. Gray's Anatomy. 38th ed. Edinburgh: Churchill Livingstone;1995:1892

25. Noltes ME, Coester AM, van der Horst-Schrivers AN, et al. Localization of parathyroid adenomas using 11C-methionine PET after prior inconclusive imaging. Langenbecks Arch Surg 2017 Jan 14. [Epub ahead of print] CrossRef Medline

26. LoPinto M, Rubio GA, Khan ZF, et al. Location of abnormal parathyroid glands: lessons from $\mathbf{8 1 0}$ parathyroidectomies. J Surg Res 2017;207:22-26 CrossRef Medline 